\title{
Dynamic Model and Motion Control Analysis of Three-link Gymnastic Robot on Horizontal Bar
}

\author{
Xie Jian, Li Zushu \\ Institute of Intelligent Automation, Chongqing University, 400044 \\ Institute of Artificial Intelligence System, Chongqing Institute of Thechnology, 400050 \\ Xjane7441@sina.com,zushuli@vip.sina.com
}

\begin{abstract}
Based on the observation of gymnast's swing-up movement, a simplified three-link horizontal bar robot is designed. With corresponding mathematic model developed, its dynamic characteristics are analyzed and simulation results are presented. From a general control perspective, an effective approach, that combines Human simulated intelligent control method with Schema theory, and its feasibility are pointed out to obtain the planning motion control.
\end{abstract}

\section{Introduction}

Through training and repeated practice, a gymnast can complete some complicated and skillful motions on gymnastic appliances such as body's curling, giant swing, swing-up and handstand, etc. Meanwhile, magnitude and direction of forces generated by body joints are harmonized and controlled with very high skills and sensory-motor intelligence. Therefore, study and understand their body movement and related joint's action are very helpful to develop the intelligent control theory for autonomous robots motion control.

As a major mechanism for studying such kind of motion that simulating human on the gymnastic apparatus, gymnastic robots, including rings [1] and horizontal bar gymnastic robots, have been proposed for many years, and some significant results can be found. Acrobot, a twodegree-of-freedom planar robot with a single actuator at its coxa, but no actuator at the wrist is discussed in [2 5], in which fuzzy control, heuristic control, partial feedback linearization and control method based on energy are applied respectively to finish planning trajectories of swing-up and inverted equilibrium.

According to the analysis of gymnast's skills and sensorymotor intelligence, however, the gymnastic robot model with only two joints is not enough to demonstrate human movement on the horizontal bar because it is apparent that athlete's shoulder joints play a very important role in controlling trunk's action. Briefly, in the process of body swing-up and being inverted, the shoulder can bend so that certain angle is formed between arms and trunk, and with torque cooperation from the coxa, this predetermined motion can be finished accurately. Certainly, on the point of bionics, human joints have no ability to drive a free 360 degree rotation, which are under the limits of strength and angle. Therefore, all these limiting factors should be represented in the gymnastic robot for obtaining more accurate physical model.

In this paper, horizontal bar gymnastic robot, with three links, including two active joints and a passive joint, is presented, and its mathematic model is derived in details, further, two kinds of typical motion behaviors, free move and forced move, are simulated in the section II. Considering conditions of torque strength and angular limits, in section III, we demonstrate a set of complete trajectory parameters analysis based on the gymnast swingup and handstand on the horizontal bar. Finally, a control method with integration of Human-Simulated Intelligent Control method and Schema Theory of Cognitive Science is proposed.

\section{Three-link gymnastic robot dynamics}

Fig. 1 shows the two-dimensional coordinates map for the horizontal bar gymnastic robot, in which the model consists of three links and three joints. Shoulder and coxa are active limited joints with rotation range of $-5 \sim 225$ degrees for the angle between arm and trunk, and $-5 \sim 180$ degrees for the angle between leg and trunk; the pivot connecting hand and bar is passive joint, which has $0 \sim 360$ degrees rotation range.

Assuming $\boldsymbol{L}_{\boldsymbol{i}}, \boldsymbol{l}_{\boldsymbol{i}}, \boldsymbol{m}_{\boldsymbol{i}}, \boldsymbol{J}_{\boldsymbol{i}}$ and $\theta_{\boldsymbol{i}}$ are respectively link length, distance from the centroid to corresponding axis, mass, moment of inertia computed from three link centroid, and angle deviated from vertical direction clockwise, where, notation $i=1,2,3$ represents three links respectively. Besides, the motors mounted on the two active joints have masses $\boldsymbol{m} \boldsymbol{c}_{1}$ and $\boldsymbol{m} \boldsymbol{c}_{2}$ respectively, and the frictional torque coefficients of three joints are notated as $c_{1}, c_{2}$ and $c_{3} . \boldsymbol{u}_{1}$ and $\boldsymbol{u}_{2}$ are symbolized as torques from two active joints which clockwise rotation is assumed to be positive. 


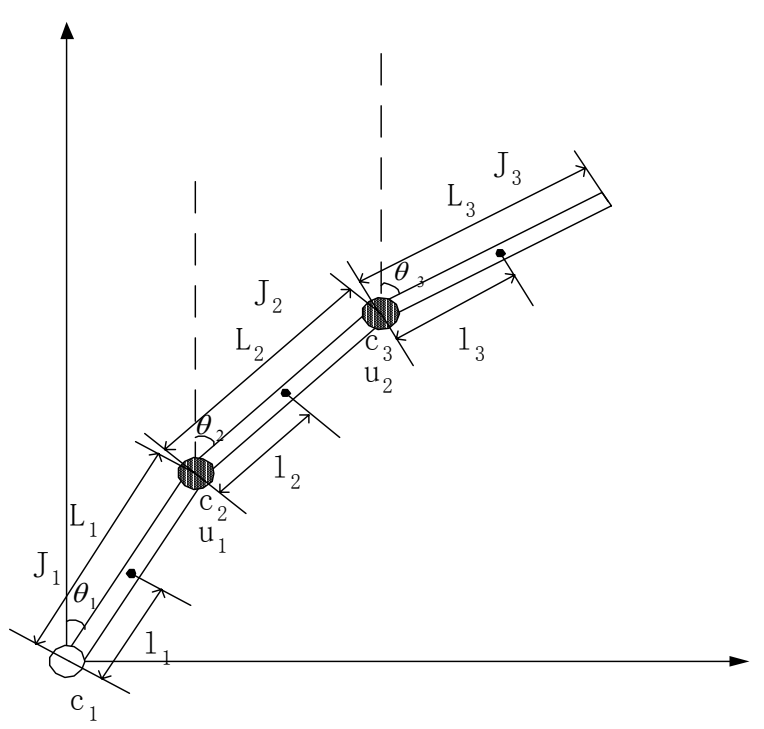

Fig.1 Coordinates map for three-link horizontal bar gymnastic robot

Then, the Lagrange equation for this model can be expressed as

$$
\frac{d}{d t}\left(\frac{\partial T}{\partial \dot{X}_{i}}\right)-\frac{\partial T}{\partial X_{i}}+\frac{\partial V}{\partial X_{i}}+\frac{\partial D}{\partial \dot{X}_{i}}=U_{i} \quad i=1,2,3
$$

where,

$$
X=\left[\begin{array}{lll}
\theta_{1} & \theta_{2} & \theta_{3}
\end{array}\right]^{T}, U=\left[\begin{array}{lll}
-u_{1} & u_{1}-u_{2} & u_{2}
\end{array}\right]^{T} ;
$$

The system kinetic energy $\boldsymbol{T}$ under the generalized coordinates, the potential function $\boldsymbol{V}$ and the energy dissipated $\boldsymbol{D}$ by joint frictions can be obtained through the equations (2), (3) and (4) respectively:

$$
\begin{aligned}
T & =\frac{1}{2} \sum_{i=1}^{3}\left[\begin{array}{l}
J_{i} \dot{\theta}_{i}^{2}+m_{i}\left[\left(\frac{d}{d t}\left(\sum_{k=i-2}^{i-1} L_{k} \sin \theta_{k}+l_{i} \sin \theta_{i}\right)\right)^{2}\right] \\
\left.+\left(\frac{d}{d t}\left(\sum_{k=i-2}^{i-1} L_{k} \cos \theta_{k}+l_{i} \cos \theta_{i}\right)\right)^{2}\right]
\end{array}\right] \\
& +\frac{1}{2} \sum_{i=1}^{2} m c_{i}\left[\begin{array}{l}
\left.\left(\frac{d}{d t} \sum_{k=i-1}^{i} L_{k} \cos \theta_{k}\right)^{2}\right] \\
\left.+\left(\frac{d}{d t} \sum_{k=i-1}^{i} L_{k} \sin \theta_{k}\right)^{2}\right]
\end{array}\right. \\
V= & \sum_{i=1}^{3} m_{i} g\left(l_{i} \cos \theta_{i}+\sum_{k=i-2}^{i-1} L_{k} \cos \theta_{k}\right) \\
& +\sum_{i=1}^{2} m c_{i} g\left(\sum_{k=i-1}^{i} L_{k} \cos \theta_{k}\right)
\end{aligned}
$$




\begin{tabular}{|c|l|l|c|l|l|}
\hline Variable & Value & Unit & Variable & Value & Unit \\
\hline $\mathrm{L}_{1}$ & 0.25 & $\mathrm{~m}$ & $\mathrm{mc}_{1}$ & 0.3 & $\mathrm{~kg}$ \\
\hline $\mathrm{L}_{2}$ & 0.25 & $\mathrm{~m}$ & $\mathrm{mc}_{2}$ & 0.3 & $\mathrm{~kg}$ \\
\hline $\mathrm{L}_{3}$ & 0.5 & $\mathrm{~m}$ & $\mathrm{~J}_{1}$ & 0.0097395 & $\mathrm{kgm}^{2}$ \\
\hline $\mathrm{l}_{1}$ & 0.125 & $\mathrm{~m}$ & $\mathrm{~J}_{2}$ & 0.011458 & $\mathrm{kgm}^{2}$ \\
\hline $\mathrm{l}_{2}$ & 0.125 & $\mathrm{~m}$ & $\mathrm{~J}_{3}$ & 0.045833 & $\mathrm{kgm}^{2}$ \\
\hline $\mathrm{l}_{3}$ & 0.25 & $\mathrm{~m}$ & $\mathrm{c}_{1}$ & 0.7056 & $\mathrm{Nms}$ \\
\hline $\mathrm{m}_{1}$ & 1.87 & $\mathrm{~kg}$ & $\mathrm{c}_{2}$ & 0.7056 & $\mathrm{Nms}$ \\
\hline $\mathrm{m}_{2}$ & 2.2 & $\mathrm{~kg}$ & $\mathrm{c}_{3}$ & 0.7056 & $\mathrm{Nms}$ \\
\hline $\mathrm{m}_{3}$ & 2.2 & $\mathrm{~kg}$ & & & \\
\hline
\end{tabular}

Table 1 Model physical parameters of three-link horizontal bar gymnastic robot

To observe the free motion behavior of this nonlinear system, we initialize three angles and no torque is executed. As shown in Figure 2 (a) (c), due to the effects of gravity and frictions, each of three links is stabilized gradually at the position pointing to the ground $\left(\theta_{1}=\theta_{2}=\theta_{3}=\pi\right)$ after damping oscillations. But, they do not swing down independently like a single pendulum because of the strong coupling among the joints. For example, when initial values for three angles are $\theta_{1}=110^{\circ}, \theta_{2}=\theta_{3}=170^{\circ}$ respectively, $\theta_{2}, \theta_{3}$ should increase to $\pi$ firstly then continue increasing if only their own gravity and joint friction applied. However, because the gravity of first link (arm) generates much stronger torque

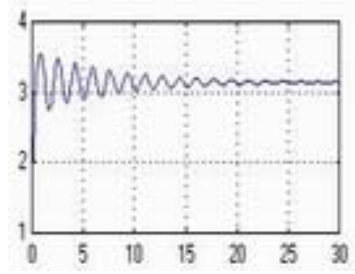

(a) $\theta_{1}(\mathrm{rad}) \mathrm{vs}$. time $(\mathrm{sec})$

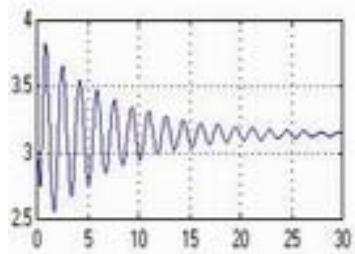

(c) $\theta_{3}(\mathrm{rad}) \mathrm{vs}$. time $(\mathrm{sec})$

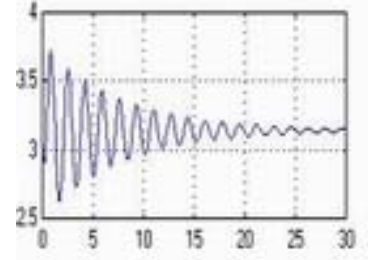

(b) $\theta_{2}(\mathrm{rad})$ vs. time (sec)

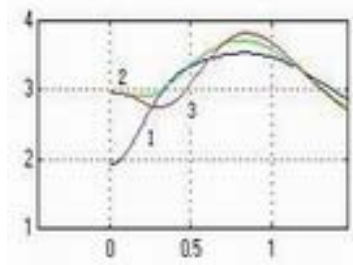

(d) $1: \theta_{1} 2: \theta_{2} 3: \theta_{3}(\mathrm{rad}) \mathrm{vs}$. time(sec)

Fig. 2 Free movement of horizontal bar gymnastic robot

than others resulting from this set of initial values, it obtains the bigger angular acceleration initially so that the $2^{\text {nd }}$ and $3^{\text {rd }}$ links (trunk and legs) have reverse acceleration.
Their trajectories are shown in Fig. 2 (d), in which unlike the first link, the $2^{\text {nd }}$ and $3^{\text {rd }}$ links move backward. The values of $\theta_{2}$ and $\theta_{3}$ are decreased at first then increased.

Furthermore, with a torque impulse executed on active joint, the robot has an initial velocity; still, each of three links is stabilized gradually at the position pointing to the ground after damping oscillations. Fig. 3 (a) (c) show the varying process of the three angles while the torque drive $\mathrm{u}_{2}=1$ occurs from $\mathrm{t}=0.1 \mathrm{~s}$ to $1.1 \mathrm{~s}$. By the coupling between the joints, $\mathrm{u}_{2}$ not only lets the $3^{\text {rd }}$ link obtain an initial velocity, but also affects the first two links. But this positive (clockwise) torque $\mathrm{u}_{2}$ effects on the $2^{\text {nd }}$ link (trunk) negatively, and due to the $\mathrm{u}_{2}$ is greater than the gravity torque of the $2^{\text {nd }}$ link, $\theta_{2}$ decreases during the period of execution of $\mathrm{u}_{2}$, until $\mathrm{u}_{2}$ stops. In contrast, the $1^{\text {st }}$ link can obtain a positive torque from the anticlockwise rotation of the $2^{\text {nd }}$ link acting on the $2^{\text {nd }}$ joint (shoulder). But this torque is small so that after a certain point the angle decreases because of gravity. Until $\mathrm{u}_{2}$ vanishes and the $2^{\text {nd }}$ link begins to rotate clockwise, the angle of the $1^{\text {st }}$ link increases (Figure 3 (d)).

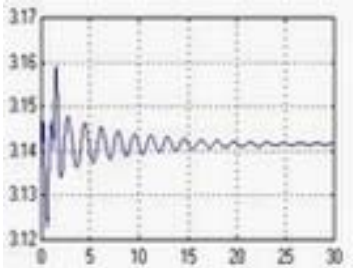

(a) $\theta_{1}$ (rad) vs. time (sec)



(c) $\theta_{3}(\mathrm{rad})$ vs. time (sec)

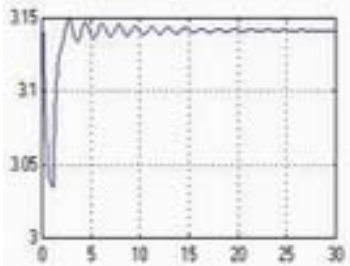

(b) $\theta_{2}(\mathrm{rad})$ vs. time $(\mathrm{sec})$

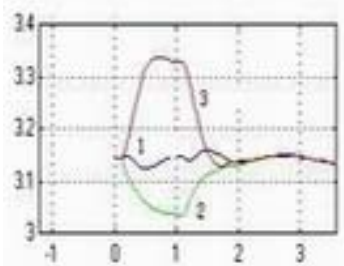

(d) $1: \theta_{1} 2: \theta_{2} 3: \theta_{3}(\mathrm{rad}) \mathrm{vs}$. time (sec)
Fig. 3 Forced movement of horizontal bar gymnastic robot

\section{Planning Motion}

In this paper, the concentrate planning motion for the control-limited three-link horizontal bar gymnastic robot is swing-up and inverted equilibrium, which can be described by means of characteristic states or energy transfer process of the system. To illustrate the system performance in this planning trajectory, we get the system state variable $s=\left(\theta_{1}, \theta_{2}, \theta_{3}, \dot{\theta}_{1}, \dot{\theta}_{2}, \dot{\theta}_{3}\right)^{T} \quad$ and energy $E=\left[\begin{array}{llll}E_{k} & E_{p 1} & E_{p 2} & E_{p 3}\end{array}\right]$, so, the motion control requirement can be defined as: 
$x_{0}=(\pi, \pi, \pi, 0,0,0) \stackrel{\text { control }}{\longrightarrow} x_{T}=(0,0,0,0,0,0)$

or

$E_{0}=\left[\begin{array}{llll}0 & 0 & 0 & 0\end{array}\right] \stackrel{\text { control work contribusin }}{\longrightarrow}$

$E_{T}=\left[\begin{array}{llll}0 & 2 g\left(m_{1} l_{1}+m c_{1} L_{1}\right) & 2 g\left(m_{2}\left(l_{2}+L\right)+m c_{2}\left(L_{1}+L_{2}\right)\right) & 2 g m_{3}\left(l_{3}+L_{1}+L_{2}\right)\end{array}\right]$

Where, $\boldsymbol{E}_{\boldsymbol{k}}$ is the system total kinetic energy (rotation energy added), and $\boldsymbol{E}_{\boldsymbol{p} 1}, \boldsymbol{E}_{\boldsymbol{p} 2}$ and $\boldsymbol{E}_{\boldsymbol{p} 3}$ are the potential energy of each link (including motors in joints) respectively.

The swing-up and inverted equilibrium control of horizontal bar gymnastic robot, studied in this paper, makes the gymnastic robot swing up from the stable equilibrium to the unstable fix point of this dynamic system, which does simulate the gymnast's motions. The control approach focuses on final accurate orientation for system states rather than specific values of the state and energy variables in the whole motion process. We can promote a high efficient method to reach the object position, if we study the moving and control skills used by the gymnast in finishing the same target.

Figure $4(\mathrm{a} \sim 1)$ refers to a typical trajectory scheme of swing-up and inverted equilibrium motion usually performed by a gymnast in the game.



Fig.4 trajectory scheme of swing-up and inverted equilibrium motion

Beginning with swinging a small angle backward from the hang position ( $\left.\theta_{1}=\theta_{2}=\theta_{3}=\pi\right)$, through driving legs or legs and the trunk $\left(\theta_{1}=\theta_{2}=\theta_{3}=\pi-\Delta \theta_{1}\right)$, the gymnast obtains certain potential energy. In this step, initial negative torque is generated from shoulders and coxa joints, but it is unnecessary to keep the three parts in line.

And then, the gymnast raises the legs forward dramatically ( $\left.\theta_{1}=\theta_{2}=\pi-\Delta \theta_{1}, \theta_{3}=\pi+\Delta \theta_{2}\right)$, driven by the coax. Further, the shoulders execute the torque to draw up the trunk following the legs so that the legs and the trunk are almost upright. At the same time the arms also lift, driven by the other two parts $\left(\theta_{1}=3 \pi / 2, \theta_{2}=\theta_{3}=2 \pi-\Delta \theta_{3}\right)$.
Due to the torque strength limited, in general, the first swing cannot reach the inverted position. But the higher potential energy is obtained, which can be transferred to be kinetic energy. Then, by the torques from shoulders and coxa, the gymnast opens the body to a line for the purpose of swinging downward (shown in Fig. $4(\mathrm{c} \sim \mathrm{g})$ ). The positive torque from the coxa should be larger than that shoulders provide, which leads the leg's angular velocity greater than trunk's $\left(\omega_{3}>\omega_{2}\right)$ and the angle between them decreases (see Fig.4 $(\mathrm{c} \sim \mathrm{d})$ ). However, the leg angular velocity $\omega_{3}$ must become less $\left(\omega_{3}<\omega_{2}\right)$ for adjusting the three moving parts in straight $\left(\theta_{1}=\theta_{2}=\theta_{3}=3 \pi / 2+\Delta \theta_{4}\right)$ (see Fig. $4(\mathrm{~d} \sim \mathrm{e})$ ).

With assistance of gravity, the gymnast swings down and continues rotating anticlockwise after passing the lowest point to obtain certain velocity $\left(\theta_{1}=\theta_{2}=\theta_{3}=\pi-\Delta \theta_{4}\right)$.

Afterwards, the reverse torque of $\mathrm{u}_{1}$ from shoulders causes the arms to be raised higher, and the angle $\theta_{2}$ formed by arms and the trunk would increase firstly then decrease because of the difference between the torque strength from shoulder and coxa. Similar transition would occur at the angle $\theta_{3}$ between the leg and trunk. Both two angles $\theta_{2}, \theta_{3}$ vary because the legs and trunk rotate faster than arms do, and finally, the whole body is stabilized at the inverted equilibrium position in straight (Figure 4 (g) to (1)).

So far, we know that the relation between the strength and direction of driving torques is highly dependent on the three angles and their speeds, which means that control strategy must be switched during the motion shifts.

\section{Indeterminable of Motion Control and Description of Control Strategy}

Horizontal bar gymnastic robot is a typical underactuated mechanical nonlinear system with fewer actuators than degrees of freedom. From a mathematic perspective, it consists of higher state dimensions than control input dimensions in generalized coordinates. In general, this kind of systems usually exhibits some features like complex internal dynamics, nonholonomic behavior, and lack of feedback linearization. Particularly, in our case, swing-up and inverted equilibrium of three-link gymnastic robot is not a simple transformation of several variables but a series behaviors and their connection. It involves cooperation among multiple control inputs and controlled variables. It requires the effective real time response and large numbers of real-time data processed. Hence, horizontal bar robot is a typical complex system. 
Compared with car-inverted pendulum, another frequently studied nonlinear dynamic system, both of them have largerange unstable motion and their energies are accumulated by dramatic vibration. There are strong coupling in both systems, which help to implement control as well as to make the system unmanageable. However, there is an apparent difference between them.

The only drive in the car-pendulum system is the horizontal driving force generated from the car. The motion control of pendulum is completed by the weight of pendulum and the coupling between pendulum and car. Therefore, two control areas can be divided clearly by the position of pendulum being up or below the horizontal line, where, direction of pendulum acceleration, driven by the car moving, is opposite.

Unlike horizontal car moving, the driving torque for horizontal bar gymnastic robot comes from two active joints, which results in a more complicated control strategy requested. From the analysis in section III, we know that both angles at two active joints among these three links have to be concerned when we analyze torque direction, strength, and angular acceleration of each link. So, it is infeasible to establish sectional control just depending on angles. Gymnastic robot finishes swing-up and inverted equilibrium on horizontal bar through a series movement, in which one action or sequential actions is for reaching a step target, such as increasing or decreasing kinetic or potential energy, and angular velocity or acceleration to a certain value. We can specify series of motions as several sections, and each section has a predetermined control object, strategy and interfaces between them. Therefore, swing-up and inverted equilibrium motion control is a parallel and series combined complicated process for horizontal bar gymnastic robot, where control relation between each section is in series, but control of motions inside each section are in both series and parallel.

Schema theory derived from the cognitive science provides an effective method to process large-scale systemic complex. Through the works by Authors in [6] [7], in which they build this method with fuzzy control or neural network to control mobile robot or autonomous robot, it proves that an approach with integration of intelligent control method and schema theory is feasible to improve control performance for complex dynamic system. Human-Simulated Intelligent Controller has been applied to the pendulum under limited torque, single and twolinked car inverted pendulum successfully [8 10], which has big advantages on simplicity and applicability for solving problems in strong coupling nonlinear system. Therefore, after simulating sensory-motor intelligence exhibited in human brain and establishing serial motion transition schema, we expect to finish planning motion control for the three-link horizontal bar gymnastic robot by a new control strategy based on Human-Simulated
Intelligent Controller and Schema Theory. This work is in the progress and will be presented in the future article.

\section{Conclusion:}

In conclusion, a three-link horizontal bar gymnastic robot has been designed and its mathematic model developed. Simulation shows the feasibility of this model. Also, a control strategy is proposed based on analyzing gymnast's motion control on horizontal bar.

This work is supported by the funds of National Natural Science Foundation of China. The number of the project is 60274022 .

\section{References}

[1] T. Yamada, K. Watanabe, K. Kiguchi and K. Izumi, "Acquiring Performance Skill of Backward Giant Circle by a Rings Gymnastic Robot", Proc. of IEEE International Conference on Robotics and Automation (ICRA 2002), pp. 1565-1570, May 11-15, 2002, Washington D.C., USA .

[2] Spong, M.W., "The Swing Up Control Problem for The Acrobot”, IEEE Control System Magazine, vol.15, no.1, 1995 pp.49-55.

[3] Spong, M.W., "Swing Up Control of the Acrobot", 1994 IEEE Int. Conf. on Robotics and Automation, San Diego, CA, pp. 2356-2361, May 8-13, 1994.

[4] Lai Xuzhi and Cai Zixing, "A Fuzzy Control Strategy for The Acrobot", Control Theory and Application No.3 Vol.17 2000, pp.326-330.

[5] H. Kajiwara, Y. Hashimoto, T. Matsuda and T. Tsuchiya, "Mathematical Analysis and Motion Control for Horizontal Bar Gymnastic Robot”, JRSJ Vol.18, No.4, pp.53-58.

[6] A. Weitzenfeld, et al, "A Neural Schema Architecture for Autonomous Robots", Proc. of 1998 International Symposium on Robotics and Automation, December 12-14, Saltillo, Coahuila, Mexico.

[7] Edy Bertolissi and Robert Holton, "Combining Schema Theory with Fuzzy Logic to Control a Mobile Robot", Proc. of the IAS 4, Karlsruhe, Germany, 27-30 March 1995 Included in the book: Intelligent Autonomous Systems, U. Rembold, R. Dillmann, L.O. Hertzberger and T. Kanade Editors, IOS Press, 1995, pp. 538-544.

[8] (in Chinese) Li Zushu, "Swing-up and Inverted Equilibrium Control for pendulum under limited torque--- Application of Human-Simulated Intelligent Controller to Nonlinear System", Control Theory and Application Vol.16, No.2, April, 1999, pp225-229.

[9] (in Chinese) Li Zushu and Chen Qingchun, "The swing up positional control of car-pendulum system", China Intelligent Automation Conference, 1998, pp513-518.

[10] (in Chinese) Zeng Ji, "Swinging up and Handstand Control Based on Human-simulated Intelligence for Cart-Two Links Pendulum System", Master Dissertation , Chongqing University, 2001.

[11] (in Chinese) Cai Zixing, "Robotics(book style)", Tsinghua University Press. 